\title{
Propriedades psicométricas de uma escala de autoeficácia para a prática de atividade física em adultos brasileiros
}

\author{
Cassiano Ricardo Rech, ${ }^{1}$ Tais Taiana Sarabia, ${ }^{2}$ \\ Rogério César Fermino, ${ }^{3}$ Pedro Curi Hallal ${ }^{4}$ \\ e Rodrigo Siqueira Reis ${ }^{3}$
}

Como citar Rech CR, Sarabia TT, Fermino RC, Hallal PC, Reis RS. Propriedades psicométricas de uma escala de autoeficácia para a prática de atividade física em adultos brasileiros. Rev Panam Salud Publica. 2011: 29(4):259-66.

RESUMO Objetivo. Verificar a validade e a fidedignidade de uma escala de autoeficácia para a prática de atividade física $(A F)$ em adultos brasileiros.

Métodos. A escala de autoeficácia foi aplicada juntamente com um questionário multidimensional face a face em 1418 indivíduos (63,4\% mulheres) com idade $\geq 18$ anos. O instrumento foi submetido à análise de validade (fatorial e construto) e fidedignidade (consistência interna e estabilidade temporal). O procedimento de teste-reteste foi aplicado em 74 indivíduos para verificar a estabilidade temporal do instrumento.

Resultados. A análise fatorial exploratória apresentou dois fatores distintos: autoeficácia para a prática de caminhada e autoeficácia para a prática de AF moderada e vigorosa (AFMV). Ambos os fatores explicaram $65,4 \%$ da variância total da escala $(20,9$ e $44,5 \%$ para caminhada e AFMV, respectivamente). Foram obtidos valores de $\alpha=0,83$ (caminhada) e 0,90 (AFMV), que indicam elevada consistência interna. Ambos os fatores da escala apresentaram correlação positiva e significativa ( $r h o \geq 0,17 ; \mathrm{P}<0,001$ ) com os indicadores de qualidade de vida (percepção de saúde, satisfação pessoal e disposição para as atividades diárias), indicando a adequada validade de construto do instrumento.

Conclusões. A escala aplicada apresenta validade, consistência interna e reprodutibilidade adequadas para avaliar a autoeficácia para a prática de AF em adultos brasileiros.

Palavras-chave Atividade motora; exercício; psicometria; auto-eficácia; Brasil.

A promoção da atividade física (AF) é uma das mais importantes estratégias

\footnotetext{
1 Universidade Estadual de Ponta Grossa, PR, Brasil; Grupo de Pesquisa em Atividade Física e Qualidade de Vida (GPAQ), Curitiba, PR, Brasil.

2 PUCPR, GPAQ, Curitiba, PR, Brasil.

3 UFPR, Programa de Pós-Graduação em Educação Física; PUCPR, GPAQ, Curitiba, PR, Brasil. Correspondência: Rodrigo Siqueira Reis, reis.rodrigo@pucpr.br

4 Universidade Federal de Pelotas (UFPel), Programa de Pós-Graduação em Educação Física, Pelotas, RS, Brasil.
}

para reduzir o risco de doenças cardiovasculares e alguns tipos de câncer em adultos (1). A prática de AF proporciona melhorias na saúde (2) e qualidade vida (3) em todas as idades. Atualmente, um dos maiores desafios dos gestores públicos é a promoção de programas de intervenção na comunidade para aumentar a prática regular de $\mathrm{AF}$ (4).

Investigações conduzidas na Europa e nos Estados Unidos demonstram que os programas de promoção da saúde baseados em mudanças de comportamento devem considerar a multidimensionalidade dos comportamentos individuais $(5,6)$, sendo que diversos fatores podem influenciar a adoção e a manutenção do comportamento ativo $(6,7)$. A teoria social cognitiva aponta a autoeficácia - o grau de confiança/crença que o indivíduo deposita na realização de determinada atividade ou na mudança de com- 
portamento - como uma das variáveis importantes nesse processo de mudança de comportamento (8).

A relação entre a autoeficácia e a prática de AF foi verificada em estudos com adolescentes (9-11), adultos (12) e idosos (13). Os resultados mostram que indivíduos com maior autoeficácia apresentam maiores níveis de AF. Além disso, existem evidências de que indivíduos com maior autoeficácia apresentam maior aderência a programas de promoção da AF (14-16). Assim, esses programas necessitam considerar a autoeficácia como uma importante variável na implementação e estruturação das atividades.

Diversos instrumentos para a avaliação da autoeficácia foram propostos na literatura (17). Entretanto, a utilização de medidas globais (que medem a autoeficácia em situações gerais da vida diária, e não especificamente em situações de AF) pode não representar esse construto de maneira coerente no contexto da AF (18). Por essa razão, algumas escalas específicas de autoeficácia para a AF foram desenvolvidas; ao menos duas são frequentemente utilizadas em estudos internacionais conduzidos em países de renda elevada $(19,20)$.

De maneira geral, as escalas avaliam a percepção do indivíduo acerca de situações intrínsecas (intraindivíduo) e extrínsecas (intergrupos) relativas à capacidade e à confiança em realizar $\mathrm{AF}$, sem considerar o tipo e a intensidade da atividade. Contudo, é possível presumir que a autoeficácia possa ser diferente dependendo do tipo e da intensidade da AF (como, por exemplo, a caminhada ou a prática de exercícios estruturados ou de intensidade mais elevada) (18). Nesse sentido, a crença na capacidade de uma determinada atividade ou na adoção de um comportamento pode ser mediada pelo grau de confiança do indivíduo na sua crença pessoal (intrínseca), a qual é, em alguma medida, dependente da interação com outros indivíduos e com o ambiente (extrínseca) (21). Por exemplo, a opinião ou a avaliação que outras pessoas manifestam sobre determinada conduta podem reforçar ou inibir a realização dessa conduta e, por consequência, aumentar ou diminuir a percepção de competência em sua execução.

Não existem estudos que tenham verificado as propriedades psicométricas dos instrumentos para a avaliação da autoeficácia no contexto latino-americano, espe- cificamente na população adulta do Brasil. Portanto, o objetivo deste estudo foi verificar a validade e a fidedignidade de uma escala de avaliação da autoeficácia para a prática de $\mathrm{AF}$ em adultos brasileiros.

\section{MATERIAIS E MÉTODOS}

\section{Delineamento do estudo}

Os dados utilizados no presente estudo foram extraídos do projeto intitulado "Nível de atividade física em adultos: associação com o ambiente percebido e suporte social". O objetivo do projeto foi avaliar aspectos de saúde, estilo de vida e hábitos de lazer de indivíduos adultos residentes no entorno de parques e praças da Cidade de Curitiba, Estado do Paraná, no Sul do Brasil. Os parques e praças foram selecionados de acordo com as condições socioeconômicas e a qualidade do ambiente para a prática de AF. Informações detalhadas acerca da seleção dos locais de coleta foram apresentadas em publicações prévias (22).

Foram considerados elegíveis os indivíduos com idade $\geq 18$ anos, residentes no entorno dos parques e praças e que moravam nos domicílios selecionados por ao menos 1 ano. Foram excluídos os indivíduos que não residiam no domicílio sorteado (exemplo: empregada doméstica, visitantes, etc.), aqueles com alguma limitação física que impedisse a prática de $\mathrm{AF}$ ou com alguma limitação cognitiva para o entendimento das questões do estudo.

Todos os 1899 segmentos de rua em um raio de 500 metros no entorno dos parques e praças foram visitados, assim como todos os domicílios identificados, com o intuito de estabelecer a representatividade amostral de moradores. Foram considerados elegíveis apenas os 1538 segmentos que continham ao menos uma residência. Após a realização do arrolamento dos segmentos e da identificação dos segmentos elegíveis, foi gerada, com o auxílio do programa Epi Info, uma tabela de números aleatórios para o sorteio de um domicílio em cada segmento. No domicílio identificado os entrevistadores foram orientados a realizar o sorteio aleatório dos moradores com base na quantidade de indivíduos elegíveis seguindo a metodologia proposta por Kish (23).

Os indivíduos participaram de maneira voluntária do estudo e foram respeitadas as orientações das Diretrizes e
Normas Regulamentadoras de Pesquisas Envolvendo Seres Humanos (resolução $\mathrm{n}^{\circ}$ 196, de 10 de outubro de 1996) do Conselho Nacional de Saúde. O estudo foi aprovado pelo Comitê de Ética em Pesquisa da Escola Superior de Educação Física da Universidade Federal de Pelotas, no Rio Grande do Sul. Os dados foram coletados entre abril e julho de 2009.

\section{Coleta de dados}

Para a coleta de dados, 25 entrevistadoras, com idade $\geq 18$ anos e ensino médio completo, receberam treinamento teórico/prático de 30 horas (5 dias) para aplicar, preencher e codificar o questionário. As entrevistadoras foram instruídas a conduzir as entrevistas com os indivíduos sorteados em ambiente separado na residência, para evitar a influência de membros da família. As entrevistas foram conduzidas em 1461 (95\%) dos 1538 segmentos elegíveis para a pesquisa. A taxa de recusa para a participação foi de 7,9\% (121 indivíduos). Para controle de qualidade das medidas foi aplicada uma reentrevista em 12,5\% da amostra. $\mathrm{Na}$ análise final do estudo, foram excluídos os indivíduos com idade $>65$ anos ou que não responderam o questionário de maneira adequada. Assim, a amostra foi constituída por 1418 indivíduos (tabela 1). Optamos por não incluir os idosos na análise por considerar que a percepção de confiança para realizar AF nesse grupo difere daquela apresentada por adultos. Consideramos ainda que, entre idosos, outros fatores, como condição de saúde e independência, podem determinar a percepção de confiança para realizar atividades físicas, sendo que esses fatores não foram considerados neste instrumento.

A fidedignidade foi testada por meio de reentrevistas em uma subamostra selecionada de maneira aleatória e sistemática entre os participantes da primeira etapa da coleta. Para tanto, os sujeitos foram convidados a participar da segunda entrevista, sendo que 74 sujeitos aceitaram participar. A segunda entrevista ocorreu após um intervalo de 7 a 10 dias e foi realizada via telefone.

\section{Medida de autoeficácia}

A avaliação da autoeficácia para a prática de AF utilizou os itens de uma escala que investiga a autoeficácia para a prática 
TABELA 1. Características descritivas dos participantes do estudo sobre autoeficácia para a prática de atividade física, Curitiba, Paraná, Brasil, 2009

\begin{tabular}{|c|c|c|c|c|c|c|}
\hline \multirow[b]{2}{*}{ Variável } & \multicolumn{2}{|c|}{$\begin{array}{l}\text { Análise fatorial e } \\
\text { de validade } \\
(n=1418)\end{array}$} & \multicolumn{2}{|c|}{$\begin{array}{l}\text { Análise de } \\
\text { fidedignidade } \\
\qquad(n=74)\end{array}$} & \multirow[b]{2}{*}{$\chi^{2}$} & \multirow[b]{2}{*}{$P$} \\
\hline & No. & $\%$ & No. & $\%$ & & \\
\hline Mulheres & 899 & 63,4 & 50 & 68,5 & 0,36 & 0,54 \\
\hline \multicolumn{7}{|l|}{ Faixa etária (anos) } \\
\hline 18 a 29 & 321 & 22,6 & 13 & 18,6 & & \\
\hline 30 a 39 & 283 & 20,0 & 17 & 24,3 & & \\
\hline 40 a 49 & 330 & 23,3 & 15 & 21,4 & & \\
\hline 50 a 59 & 338 & 23,8 & 21 & 30,0 & & \\
\hline$\geq 60$ & 146 & 10,8 & 4 & 5,7 & 4,04 & 0,02 \\
\hline \multicolumn{7}{|l|}{ Nível socioeconômico } \\
\hline Elevado & 533 & 37,8 & 11 & 14,9 & & \\
\hline Intermediário & 703 & 49,9 & 38 & 51,4 & & \\
\hline Baixo & 174 & 12,3 & 25 & 33,8 & 0,16 & 0,23 \\
\hline Percepção positiva de saúde & 973 & 68,6 & 46 & 62,2 & 1,07 & 0,30 \\
\hline Satisfação pessoal & 1124 & 79,3 & 49 & 66,2 & 6,37 & 0,01 \\
\hline $\begin{array}{l}\text { Capacidade para as } \\
\text { atividades diárias }\end{array}$ & 1165 & 82.2 & 64 & 86.5 & 0.63 & 0.42 \\
\hline
\end{tabular}

de AF de intensidade moderada a vigorosa (AFMV) (24). Esse instrumento foi desenvolvido pelo grupo de trabalho da San Diego State University, nos Estados Unidos, e aplicado no Neighborhood Quality of Life Study (24). O desenvolvimento dessa escala foi baseado em escalas específicas de autoeficácia para a prática de exercício físico, as quais foram propostas por autores de referências na área $(19,20)$.

Originalmente, as duas primeiras escalas $(19,20)$ eram compostas por 18 itens relacionados à autoeficácia para a prática de AF geral, com opções de resposta dispostas em uma escala likert de cinco pontos. A escala proposta para aplicação em estudos epidemiológicos utiliza 10 itens, com uma escala likert de cinco pontos (24).

No presente estudo, a escala de autoeficácia foi testada considerando-se 10 itens (24) divididos em dois blocos, com questões específicas relacionadas à prática de caminhada ou de AFMV analisadas separadamente (ver escala no anexo 1). Optamos por analisar as características psicométricas da escala com base no tipo e intensidade da AF porque a percepção de capacidade para realizar determinada atividade é específica para cada situação (18). A caminhada é uma atividade comum ao ser humano, enquanto as AFMVs envolvem alguma complexidade de ações motoras, além de maior intensidade.

Para o presente estudo, a escala original (24) foi submetida aos procedimentos sugeridos na literatura para a tradução e adaptação transcultural de instrumentos de pesquisa $(25,26)$. A versão original foi traduzida para o português brasileiro de maneira independente por dois profissionais brasileiros e fluentes na língua inglesa. As duas traduções foram comparadas e discutidas pelos autores e, nos casos de divergências gramaticais e redação, foram realizadas modificações para se obter uma versão única que mantivesse as características fundamentais do questionário original. Posteriormente, na etapa de retrotradução (back translation), a versão em português foi traduzida para o inglês por um especialista norte-americano na área de AF que não participou da primeira etapa de tradução. As duas versões em inglês foram comparadas para verificar eventuais inconsistências $(25,26)$.

As questões traduzidas foram então aplicadas em uma amostra de 20 adultos com baixa, média e elevada escolaridade para verificar a clareza e a compreensão quanto à ordem e à forma das escalas. Ao longo das entrevistas, a escala original (cinco pontos) foi adaptada para uma escala de dois pontos (sim e não), uma vez que os entrevistados de escolaridade mais baixa apresentaram elevada dificuldade de compreensão.

Essa opção também demonstrou ser mais aplicável para estudos amplos (por exemplo: inquéritos domiciliares), nos quais o uso de escalas psicométricas complexas pode ser limitante. De fato, essa característica foi observada em estudos com emprego de questionários (27) e correlatos (28) de AF na América Latina.
Os escores da escala de autoeficácia foram computados com a soma das respostas de cada bloco (caminhada e AFMV), considerando-se a resposta "sim" como valor 1 e "não" como valor zero, originando dois escores que podem variar de zero a 5 pontos. Os escores obtidos indicam a autoeficácia, sendo que, quanto maior o valor, mais elevada é a autoeficácia para a prática de caminhada ou de AFMV.

Definiu-se como AFMV qualquer situação em que o indivíduo desempenhasse algum esforço físico que o fizesse respirar mais forte do que o normal ou que elevasse de maneira expressiva os batimentos cardíacos (por exemplo, corrida, futebol, voleibol, basquete, ginástica, natação e outros). Essas características foram determinadas através da percepção individual para cada atividade.

\section{Atividade física}

A AF foi avaliada com o módulo de lazer do International Physical Activity Questionnaire (29) versão longa, devidamente traduzido e validado para a população brasileira (30). As questões referiam-se a frequência semanal e duração em minutos das caminhadas ou AFMV (sendo o mínimo 10 minutos). A AFMV foi agrupada e analisada separadamente da caminhada para que as questões da escala de autoeficácia pudessem ser analisadas de maneira específica.

\section{Variáveis demográficas e de qualidade de vida}

As variáveis sexo, idade, nível socioeconômico e qualidade de vida (percepção de saúde, satisfação pessoal e capacidade para as atividades diárias) também foram avaliadas. O nível socioeconômico foi avaliado com base no Critério de Classificação Econômica Brasil, utilizado pela Associação Brasileira de Empresas de Pesquisa (ABEP) (31). Operacionalmente, o nível socioeconômico foi categorizado em três estratos, com base no agrupamento das classes econômicas: estrato alto $(\mathrm{A} 1+\mathrm{A} 2)$, intermediário $(\mathrm{B} 1+$ B2) e baixo $(\mathrm{C} 1+\mathrm{C} 2+\mathrm{D}+\mathrm{E})$. A percepção de saúde foi avaliada com a questão: "Como você considera a sua saúde?", tendo como opções de resposta uma escala likert de quatro pontos (ruim, regular, boa e muito boa). As opções "boa" e "muito boa" foram agrupadas para caracterizar a variável "per- 
cepção positiva de saúde". Também foram realizadas duas questões relativas a satisfação pessoal e capacidade para as atividades diárias (32): 1) "Você está satisfeito consigo mesmo?" e 2) "Você está satisfeito com sua capacidade de desempenhar as atividades do dia a dia?". Ambas as questões tiveram como resposta uma escala likert de cinco pontos (muito insatisfeito, insatisfeito, nem insatisfeito/nem satisfeito, satisfeito e muito satisfeito), que foram agrupadas em duas categorias: a) insatisfeito (muito insatisfeito, insatisfeito e nem insatisfeito/nem satisfeito) e b) satisfeito (satisfeito e muito satisfeito).

\section{Análise de dados}

A estrutura da escala foi analisada por análise fatorial exploratória com emprego da rotação Varimax para verificar a carga de cada item da escala. A adequação da amostra foi testada com a estatística Kaiser-Meyer-Olkin (KMO). Foram aceitos os eigenvalues $\geq 1,0$ e aqueles itens com carga $>0,4$ para definir os fatores obtidos na análise.

Para evidenciar a validade de construto da escala de autoeficácia, a soma dos escores de autoeficácia para caminhada e AFMV foi relacionada com os indicadores de qualidade de vida (percepção de saúde, satisfação pessoal e capacidade para as atividades diárias). Para tanto, foi empregada a correlação de postos ordenados de Spearman (rho). Nesta análise assume-se a hipótese de que existe uma associação positiva e específica entre os escores da escala e a percepção do indivíduo (por exemplo: o escore de autoeficácia para a caminhada versus capacidade para as atividades diárias).

Por fim, foi testada a fidedignidade através da consistência interna ( $\alpha$ de Cronbach e coeficiente de kappa) e estabilidade temporal (coeficiente de correlação intraclasse, CCI), sendo esta última pelo método teste-reteste com 7 a 10 dias de intervalo. Foram considerados adequados os valores de $\alpha$ e CCI $\geq 0,70$ para considerar a fidedignidade aceitável. Todas as análises foram realizadas no programa Statistical Package for the Social Sciences (SPSS) 15.0, com nível de significância de $5 \%$.

\section{RESULTADOS}

Na tabela 1 são apresentadas as características dos 1418 participantes do es-

TABELA 2. Análise fatorial exploratória da escala de autoeficácia para prática de atividade física em adultos da Cidade de Curitiba, Paraná, Brasil, 2009a

\begin{tabular}{|c|c|c|c|}
\hline \multirow{2}{*}{\multicolumn{2}{|c|}{ Itens da escala de autoeficácia para a prática de atividade física }} & \multicolumn{2}{|c|}{ Fatores da escala } \\
\hline & & 1 & 2 \\
\hline 1 & Você consegue fazer caminhada quando você está cansado? & 0,78 & \\
\hline 2 & Você consegue fazer caminhada quando você está de mau humor? & 0,83 & \\
\hline 3 & Você consegue fazer caminhada quando você está sem tempo? & 0,66 & \\
\hline 4 & Você consegue fazer caminhada quando você está de férias? & 0,78 & \\
\hline 5 & Você consegue fazer caminhada quando está muito frio? & 0,74 & \\
\hline 6 & Você consegue fazer AFMVb quando você está cansado? & & 0,85 \\
\hline 7 & Você consegue fazer AFMVb quando você está de mau humor? & & 0,88 \\
\hline 8 & Você consegue fazer AFMVb quando você está sem tempo? & & 0,71 \\
\hline 9 & Você consegue fazer AFMVb quando você está de férias? & & 0,82 \\
\hline 10 & Você consegue fazer AFMVb quando está muito frio? & & 0,88 \\
\hline \multicolumn{2}{|c|}{ Eigenvalue } & 2,1 & 4,4 \\
\hline \multicolumn{2}{|c|}{ \% Variância do fator } & 20,9 & 44,5 \\
\hline \multicolumn{2}{|c|}{ \% Variância total da escala } & \multicolumn{2}{|c|}{65,4} \\
\hline
\end{tabular}

TABELA 3. Análise de consistência interna dos itens da escala de autoeficácia para prática de atividade física em adultos da Cidade de Curitiba, Paraná, Brasil, 2009a

\begin{tabular}{llc}
\hline Itens da escala de autoeficácia para a prática de atividade física & $\begin{array}{c}\text { Valor de } \alpha \\
\text { caso o item } \\
\text { fosse deletado }\end{array}$ & $\begin{array}{c}\alpha \\
\text { total }^{b}\end{array}$ \\
\hline $1 \quad$ Você consegue fazer caminhada quando você está cansado? & 0,79 \\
2 Você consegue fazer caminhada quando você está de mau humor? & 0,77 \\
$3 \quad$ Você consegue fazer caminhada quando você está sem tempo? & 0,83 \\
4 Você consegue fazer caminhada quando você está de férias? & 0,78 \\
5 Você consegue fazer caminhada quando está muito frio? & 0,80 \\
6 Você consegue fazer AFMVc quando você está cansado? & 0,87 \\
7 Você consegue fazer AFMVc quando você está de mau humor? & 0,86 \\
8 Você consegue fazer AFMVc quando você está sem tempo? & 0,90 \\
9 Você consegue fazer AFMVc quando você está de férias? & 0,88 \\
10 Você consegue fazer AFMVc quando está muito frio? & 0,86 \\
\hline
\end{tabular}

1418 participantes.

b Alfa de Cronbach.

c $\mathrm{AFMV}=$ atividade física moderada e vigorosa.

tudo e da subamostra de 74 participantes utilizada para avaliação da estabilidade temporal. Na amostra total houve maior predominância de mulheres $(63,4 \%)$, de participantes com idade entre 50 e 59 anos $(23,8 \%)$ e do estrato econômico intermediário $(49,9 \%)$. Foi verificada uma elevada percepção positiva de saúde $(68,6 \%)$, satisfação pessoal $(79,3 \%)$ e capacidade para as atividades diárias $(82,2 \%)$. A subamostra apresentou características similares na maior parte das variáveis analisadas, porém incluiu um número menor de indivíduos com idade $\geq 60$ anos e apresentou percepção menos positiva de saúde.

A análise fatorial exploratória identificou dois fatores independentes para os itens que compõem a escala (eigenvalues $\geq 1,0$ ) (tabela 2). Ambos os fatores expli- cam $65,4 \%$ da variância total da escala. $\mathrm{O}$ fator 1 foi composto por cinco itens relacionados à autoeficácia para a prática da caminhada (variância $=20,9 \%$ ). O fator 2 também foi composto por cinco itens, porém relacionados à autoeficácia para a prática de AFMV (variância $=44,5 \%$ ). Todos os itens apresentaram carga $\geq 0,66$ nos respectivos fatores. A amostra apresentou tamanho adequado para os procedimentos empregados na análise fatorial $(\mathrm{KMO}=0,90 ; P<0,001)$.

A análise de consistência interna ( $\alpha$ de Cronbach) demonstrou valores significativos e superiores a 0,7 para todos os itens (tabela 3). Todos os itens mostraram-se igualmente importantes para compor o valor total e foram, portanto, mantidos na escala final. Os valores totais do $\alpha$ de Cronbach foram superiores a 0,8 tanto 
TABELA 4. Valores do coeficiente de correlação de Spearman (rho) entre os indicadores de qualidade de vida e o escore da escala de autoeficácia para a prática de atividade física em adultos da Cidade de Curitiba, Paraná, Brasil, 2009a

\begin{tabular}{lcc}
\hline Indicadores de qualidade de vida & $\begin{array}{c}\text { Autoeficácia } \\
\text { com a caminhada }\end{array}$ & $\begin{array}{c}\text { Autoeficácia } \\
\text { com a AFMV }\end{array}$ \\
\hline Percepção positiva de saúde & $0,18^{\mathrm{c}}$ & $0,19^{\mathrm{c}}$ \\
Satisfação pessoal & $0,19^{\mathrm{c}}$ & $0,19^{\mathrm{c}}$ \\
Disposição para as atividades diárias & $0,21^{\mathrm{c}}$ & $0,17^{\mathrm{c}}$ \\
\hline a 1418 participantes. & & \\
b AFMV = atividade física moderada e vigorosa. & & \\
c $P<0,001$. &
\end{tabular}

TABELA 5. Resultados de reentrevistas para testar a fidedignidade da escala de autoeficácia para prática de atividade física em adultos da Cidade de Curitiba, Paraná, Brasil, 2009a

\begin{tabular}{|c|c|c|c|c|}
\hline & Itens da escala de autoeficácia para a prática de atividade física & $\% \mathrm{C}^{\mathrm{b}}$ & kappac & $\mathrm{CCl}(\mathrm{IC} 95 \%)^{\mathrm{d}}$ \\
\hline 1 & Você consegue fazer caminhada quando você está cansado? & 75,6 & 0,51 & \\
\hline 2 & Você consegue fazer caminhada quando você está de mau humor? & 77,0 & 0,48 & $0,81^{e}$ \\
\hline 3 & Você consegue fazer caminhada quando você está sem tempo? & 74,3 & 0,48 & $(0,69$ a 0,88$)$ \\
\hline 4 & Você consegue fazer caminhada quando você está de férias? & 79,7 & 0,55 & \\
\hline 5 & Você consegue fazer caminhada quando está muito frio? & 86,5 & 0,72 & \\
\hline 6 & Você consegue fazer AFMV q quando você está cansado? & 70,0 & 0,40 & \\
\hline 7 & Você consegue fazer AFMV quando você está de mau humor? & 74,3 & 0,47 & $0,70^{e}$ \\
\hline 8 & Você consegue fazer AFMV quando você está sem tempo? & 73,0 & 0,40 & $(0,64$ a 0,77$)$ \\
\hline 9 & Você consegue fazer AFMV quando você está de férias? & 74,3 & 0,48 & \\
\hline 10 & Você consegue fazer $\mathrm{AFMV}^{\dagger}$ quando está muito frio? & 70,3 & 0,42 & \\
\hline
\end{tabular}

a 74 participantes.

b \%C = percentual de concordância (teste-reteste).

c $P<0,005$.

d $\mathrm{CCl}(\mathrm{IC} 95 \%)$ = coeficiente de correlação intraclasse (intervalo de confiança de 95\%).

$P<0,005$.

f $\mathrm{AFMV}=$ atividade física moderada e vigorosa.

para a caminhada $(\alpha=0,83)$ quanto para a $\operatorname{AFMV}(\alpha=0,90)$, demonstrando adequada consistência interna. A validade do construto foi confirmada por meio da análise da correlação entre os indicadores de qualidade de vida e os escores da escala, que revelou uma correlação significativa e positiva (tabela 4).

Os resultados das análises de estabilidade temporal são apresentados na tabela 5. A concordância foi elevada para todos os itens da escala, tanto para caminhada $(74,3$ a $86,5 \%)$ quanto para AFMV $(70,0$ a $74,3 \%)$. Ao considerar os escores totais de cada escala, os valores de CCI foram de 0,81 para caminhada (intervalo de confiança de $95 \%$, IC95\%: 0,69 a 0,88) e 0,70 para AFMV (IC95\%: 0,64 a 0,77).

\section{DISCUSSÃO}

A autoeficácia - nível de confiança do indivíduo - é um importante mediador da intenção para a prática de $\mathrm{AF}$ em adultos (7). Sendo assim, a avaliação da autoeficácia frente a um comportamento é de fundamental importância para a ela- boração de programas efetivos para a promoção do comportamento ativo. $\mathrm{O}$ presente estudo examinou as propriedades psicométricas de uma escala de avaliação da autoeficácia para a prática de $\mathrm{AF}$, considerando duas diferentes intensidades (caminhada e AFMV) em adultos. Os resultados demonstraram que, ao analisar a autoeficácia com a AF, tanto a intensidade quanto o tipo das atividades devem ser considerados, ou seja, a autoeficácia é diferente e específica para caminhada e AFMV.

De maneira geral, as escalas propostas na literatura são baseadas no conceito de exercício físico $(19,20,33)$, não considerando diferentes intensidades de $\mathrm{AF}$ como um fator que pode modificar a percepção de competência ou intenção para iniciar e manter a prática do exercício. Com o presente estudo, espera-se apresentar uma escala válida para mensurar, principalmente em estudos populacionais, o construto da autoeficácia para caminhada e AFMV.

A autoeficácia para AF é um construto multidimensional $(19,34)$. A teoria social cognitiva (18) considera a autoeficácia como o grau de confiança do indivíduo para realizar uma atividade específica. Por ser um construto que depende de tal especificidade, é possível apresentar a hipótese de que indivíduos podem simultaneamente possuir confiança suficiente para realizar a caminhada com regularidade e considerar-se menos aptos para praticar atividades mais intensas e complexas (AFMV), como corrida ou natação. Da mesma forma, as barreiras, internas e externas, relatadas para a caminhada não são as mesmas relatadas para AFMV (7), o que reforça a necessidade de distinção entre atividades de intensidade diferente. Nesse sentido, a base teórica utilizada no desenvolvimento da escala de autoeficácia (24) assume essa premissa e avalia o construto considerando diferentes intensidades de AF. Essa hipótese poderá ser melhor explorada a partir da validação da presente escala para emprego em adultos brasileiros.

A consistência interna da escala foi adequada e similar à relatada por outros estudos, tanto para caminhada $(\alpha=0,83)$ quanto para AFMV $(\alpha=0,90)$. O $\alpha>0,70$ para todos os itens da escala demonstra que todos contribuem de forma importante para a construção do fator e que a estrutura de ambas as partes da escala é consistente. Outros estudos que investigaram as características psicométricas de escalas de autoeficácia para a prática de AF encontraram valores de fidedignidade elevados em crianças $(\alpha=0,88)$ (35), adolescentes $(\alpha=0,73)$, adultos $(\alpha=$ $0,85)(19)$ e idosos ( $\alpha$ entre 0,75 e 0,92 ) $(36,37)$. Tais valores são semelhantes aos observados em nosso estudo (caminhada $\alpha=0,83$, AFMV $\alpha=0,90$ ).

A validade de construto foi analisada pela comparação entre os fatores da escala de autoeficácia e indicadores de qualidade de vida (32) (percepção de saúde, satisfação pessoal e capacidade para as atividades diárias). A literatura traz evidências claras de uma relação positiva entre os escores de autoeficácia e os indicadores de qualidade de vida $(38,39)$. No presente estudo, foram observados valores de correlação significativos e positivos entre os três indicadores de qualidade de vida empregados e os escores de autoeficácia para caminhada e AFMV, confirmando a validade de construto.

Os resultados de fidedignidade também demonstraram uma elevada concordância entre os itens $(70,0$ a $86,5 \%)$, valores de kappa significativos $(P<0,01)$ 
e um CCI elevado. Esses resultados demonstraram que a escala apresenta estabilidade temporal adequada aos parâmetros utilizados pela literatura (40), assim como outras escalas que analisaram a autoeficácia para a caminhada como deslocamento (41) ou estudos realizados em crianças (35), adolescentes (42), adultos (19) e idosos (37).

Algumas limitações devem ser consideradas para a extrapolação dos resultados encontrados. A escala foi testada em uma amostra de adultos da Cidade de Curitiba, no Sul do Brasil, os quais apresentaram elevada percepção de saúde, satisfação pessoal, capacidade para as atividades diárias e renda. Devido às diferenças econômicas e socioculturais, os pesquisadores de outras localidades devem considerar essas características ao extrapolar os resultados para os seus contextos. Até o presente momento, este é o único estudo que testou as qualidades psicométricas de uma escala de avaliação da autoeficácia para a AF em adultos brasileiros. Entretanto, é importante ressaltar que foram utilizadas medidas autorreportadas e que podem apresentar uma estimativa menos precisa da AF. É recomendável a realização de outros estudos de validade concorrente para a escala, em diferentes grupos populacionais, com distintas condições sociais e de saúde.

Entretanto, com base nos presentes resultados, pode-se concluir que a escala proposta apresenta validade, consistência interna e fidedignidade adequadas para avaliar a autoeficácia para a prática de AF em adultos brasileiros. O instrumento proposto no presente estudo contribui para que um novo fator correlato da AF seja avaliado e analisado nos programas de intervenção que se baseiam na mudança de comportamento.

Agradecimentos. CRR é bolsista da Fundação Araucária e RCF é bolsista da Coordenação de Aperfeiçoamento de Pessoal de Nível Superior (CAPES). $\mathrm{O}$ estudo recebeu financiamento do Conselho Nacional de Desenvolvimento Científico e Tecnológico (CNPq) (edital universal/MCT/CNPq 014/2008).

ANEXO 1. Escala de autoeficácia para prática de atividade física utilizada em adultos brasileiros, 2009

Escala de avaliação da autoeficácia para a prática de atividade física

As questões abaixo estão relacionadas ao quanto você se sente capaz de realizar atividade física mediante algumas condições. Não existem respostas erradas. Por favor, assinale apenas uma opção para cada questão.

Para responder as questões lembre-se que:

Atividades físicas de intensidade moderada são aquelas que precisam de algum esforço físico, que te fazem respirar um pouco mais forte que o normal e/ou que fazem o seu coração bater um pouco mais forte.

Atividades físicas de intensidade vigorosa são aquelas que precisam de um grande esforço físico, que te fazem respirar muito mais forte que o normal e/ou que fazem o seu coração bater mais forte.

Seção 1. Nesta seção considere somente a caminhada que você faz no seu tempo livre.

1. Você consegue fazer caminhada no seu tempo livre quando você está cansado?

${ }^{0}$ [ ] Não ${ }^{1}[$ ] Sim

2. Você consegue fazer caminhada no seu tempo livre quando você está de mau humor?

$$
{ }^{0} \text { [ ] Não }{ }^{1} \text { [ ] Sim }
$$

3. Você consegue fazer caminhada no seu tempo livre quando você está sem tempo?

${ }^{0}$ [ ] Não ${ }^{1}$ [ ] Sim

4. Você consegue fazer caminhada no seu tempo livre quando você está de férias?

${ }^{0}$ [ ] Não ${ }^{1}[$ ] Sim

5. Você consegue fazer caminhada no seu tempo livre quando está muito frio?

${ }^{0}$ [ ] Não ${ }^{1}[$ ] Sim
Seção 2. Nesta seção considere as atividades físicas de intensidade moderada e/ou vigorosa (AFMV) (exemplo: correr, nadar, pedalar, jogar vôlei, futebol, etc.). Não inclua a caminhada.

6. Você consegue fazer AFMV no seu tempo livre quando você está cansado?

$$
{ }^{0} \text { [ ] Não }{ }^{1} \text { [ ] Sim }
$$

7. Você consegue fazer AFMV no seu tempo livre quando você está de mau humor?

$$
{ }^{0} \text { [ ] Não }{ }^{1}[\text { ] Sim }
$$

8. Você consegue fazer AFMV no seu tempo livre quando você está sem tempo?

$$
{ }^{0}\left[\text { ] Não }{ }^{1}[\text { ] Sim }\right.
$$

9. Você consegue fazer AFMV no seu tempo livre quando você está de férias?

$$
{ }^{0} \text { [ ] Não }{ }^{1}[\text { ] Sim }
$$

10. Você consegue fazer AFMV no seu tempo livre quando está frio?
${ }^{0}$ [ ] Não ${ }^{1}$ [ ] Sim 


\section{REFERÊNCIAS}

1. U.S. Department of Health and Human Services. Physical activity guidelines advisory committee report, 2008. Washington: USDHHS; 2008. Disponível em: www. health. gov/paguidelines/Report/pdf/Committee Report.pdf. Acessado em 22 de abril de 2009.

2. Andersen LB, Schnohr P, Schroll M, Hein HO. All-cause mortality associated with physical activity during leisure time, work, sports, and cycling to work. Arch Intern Med. 2000;160(11): $1621-8$.

3. Bize R, Johnson JA, Plotnikoff RC. Physical activity level and health-related quality of life in the general adult population: a systematic review. Prev Med. 2007;45(6):401-15.

4. Sallis J, Cervero RB, Ascher W, Henderson KA, Kraft MK, Kerr J. An ecological approach to creating active living communities. Annu Rev Public Health. 2006;27:297-322.

5. Norman GJ, Zabinski MF, Adams MA, Rosenberg DE, Yaroch AL, Atienza AA. A review of eHealth interventions for physical activity and dietary behavior change. Am J Prev Med. 2007;33(4):336-45.

6. Kahn EB, Ramsey LT, Browson RC, Heath GW, Howze EH, Powell KE, et al. The effectiveness of interventions to increase physical activity. A systematic review. Am J Prev Med. 2002;22(4 Suppl):73-107.

7. Trost SG, Owen N, Bauman AE, Sallis JF, Brown W. Correlates of adults' participation in physical activity: review and update. Med Sci Sports Exerc. 2002;34(12):1996-2001.

8. Bandura AA. Social foundations of thought and action: a social cognitive theory. Englenwood Cliffs: Prentice Hall; 1986.

9. Dishman RK, Motl RW, Saunders R, Felton G, Ward DS, Dowda M, et al. Self-efficacy partially mediates the effect of a school-based physical-activity intervention among adolescent girls. Prev Med. 2004;38(5):628-36.

10. Deforche B, Van Dyck D, Verloigne M, De Bourdeaudhuij I. Perceived social and physical environmental correlates of physical activity in older adolescents and the moderating effect of self-efficacy. Prev Med. 2010; 50(Suppl 1):S24-9.

11. Van Der Horst K, Paw MJ, Twisk JW, Van Mechelen W. A brief review on correlates of physical activity and sedentariness in youth. Med Sci Sports Exerc. 2007;39(8): 1241-50.

12. Opdenacker J, Delecluse C, Boen F. The longitudinal effects of a lifestyle physical activity intervention and a structured exercise intervention on physical self-perceptions and selfesteem in older adults. J Sport Exerc Psychol. 2009;31(6):743-60.

13. McAuley E, Jerome GJ, Elavsky S, Marquez DX, Ramsey SN. Predicting long-term maintenance of physical activity in older adults. Prev Med. 2003;37(2):110-8.

14. Opdenacker J, Boen F, Vanden Auweele Y, De Bourdeaudhuij I. Effectiveness of a lifestyle physical activity intervention in a women's organization. J Womens Health (Larchmt). 2008;17(3):413-21
15. Sallis JF, Alcaraz JE, McKenzie TL, Hovell MF. Predictors of change in children's physical activity over 20 months. Variations by gender and level of adiposity. Am J Prev Med. 1999; 16(3):222-9.

16. Motl RW, Dishman RK, Ward DS, Saunders RP, Dowda M, Felton G, et al. Perceived physical environment and physical activity across one year among adolescent girls: self-efficacy as a possible mediator? J Adolesc Health. 2005;37(5):403-8.

17. Dougherty CM, Johnston SK, Thompson EA. Reliability and validity of the self-efficacy expectations and outcome expectations after implantable cardioverter defibrillator implantation scales. Appl Nurs Res. 2007;20(3):116-24.

18. Bandura A. Self-efficacy: toward a unifying theory of behavioral change. Psychol Rev. 1977;84(2):191-215.

19. Sallis JF, Pinski RB, Grossman RM, Patterson TL, Nader PR. The development of self-efficacy scales for health-related diet and exercise behaviors. Health Educ Res. 1988;3(3):283-92.

20. Bandura A. Guide for constructing selfefficacy scales. Em: Pajares F, Urdan TC, editors. Self-efficacy: beliefs of adolescents Charlotte: Information Age Publishing; 2006. Pp. 307-37.

21. Bandura A. Self-efficacy mechanism in human agency. Am Psychol. 1982;37(2): 122-47.

22. Hino AAF, Reis RS, Ribeiro IC, Parra DC, Brownson RC, Fermino RC. Using observational methods to evaluate public open spaces and physical activity in Brazil. J Phys Act Health. 2010;7(Suppl 2):S146-54.

23. Kish L. Sampling organizations and groups of unequal sizes. Am Sociol Rev. 1965;30:564-72.

24. Neighborhood Quality of Life Study Survey (NQLS). Disponível em: www. drjamessallis. sdsu.edu/Documents/NQLS_S1.pdf. Acessado em 15 de novembro de 2010

25. Guillemin F, Bomabardier C, Beaton D. Crosscultural adaptation of health-related quality of life measures: literature review and proposed guidelines. J Clin Epidemiol. 1993; 46(12):1417-32.

26. Vijver FVD, Hambleton RK. Translating tests: some practical guidelines. Eur Psychol. 1996; 1(2):89-99.

27. Hallal PC, Gomez LF, Parra DC, Lobelo F, Mosquera J, Florindo AA, et al. Lessons learned after 10 years of IPAQ use in Brazil and Colombia. J Phys Act Health. 2010; 7(Suppl 2):S259-64

28. Santos MS, Hino AAF, Reis RS, RodriguezAñez CR. Prevalência de barreiras para a prática de atividade física em adolescentes. Rev Bras Epidemiol. 2010;13(1):94-104.

29. Craig CL, Marshall AL, Sjostrom M, Bauman $\mathrm{AE}$, Booth ML, Ainsworth BE, et al. International physical activity questionnaire: 12country reliability and validity. Med Sci Sports Exerc. 2003;35(8):1381-95.

30. Matsudo SMM, Araújo TL, Matsudo VKR, Andrade DR, Andrade EL, Oliveira LC, et al. Questionário Internacional de Atividade
Física (IPAQ): estudo de validade e reprodutibilidade no Brasil. Rev Bras Ativ Fis Saude. 2001;6(2):5-17.

31. Associação Brasileira de Empresas de Pesquisa. Critério de classificação econômica do Brasil 2009 (Dados com base no Levantamento Sócio Econômico 2006 e 2007 — IBOPE). Disponível em: www.abep.org/novo/Utils/FileGenerate. ashx?id=170. Acessado em 08 de março de 2011.

32. Fleck MP, Louzada $S$, Xavier $M$, Chachamovich E, Vieira G, Santos L, et al. Aplicação da versão em português do instrumento de avaliação de qualidade de vida da Organização Mundial da Saúde (WHOQOL100). Rev Saude Publica. 1999;33(2):198-205.

33. Dwyer JJ, Allison KR, Makin S. Internal structure of a measure of self-efficacy in physical activity among high school students. Soc Sci Med. 1998;46(9):1175-82.

34. McAuley E, Jacobson L. Self-efficacy and exercise participation in sedentary adult females. Am J Health Promot. 1991;5(3):185-91.

35. Aedo A, Avila H. Nuevo cuestionario para evaluar la autoeficacia hacia la actividad física en niños. Rev Panam Salud Publica. 2009; 26(4):324-9.

36. Lee LL, Perng SJ, Ho CC, Hsu HM, Lau SC, Arthur A. A preliminary reliability and validity study of the Chinese version of the selfefficacy for exercise scale for older adults. Int J Nurs Stud. 2009;46(2):230-8.

37. Resnick B, Jenkins LS. Testing the reliability and validity of the Self-Efficacy for Exercise Scale. Nurs Res. 2000;49(3):154-9.

38. Paxton RJ, Motl RW, Aylward A, Nigg CR Physical activity and quality of life-the complementary influence of self-efficacy for physical activity and mental health difficulties. Int J Behav Med. 2010;17(4):255-63.

39. Motl RW, Snook EM. Physical activity, selfefficacy, and quality of life in multiple sclerosis. Ann Behav Med. 2008;35(1):111-5.

40. Terwee $C B$, Bot SD, de Boer MR, van der Windt DA, Knol DL, Dekker J, et al. Quality criteria were proposed for measurement properties of health status questionnaires. J Clin Epidemiol. 2007;60(1):34-42.

41. Dunton GF, Schneider M. Reliability and validity of a scale to measure self-efficacy to overcome barriers to walking for transportation. J Phys Act Health. 2006;3(Suppl 1):48-58.

42. Reynolds KD, Killen JD, Bryson SW, Maron DJ, Taylor CB, Maccoby N, et al. Psychosocial predictors of physical activity in adolescents. Prev Med. 1990;19(5):541-51.

Manuscrito recebido em 24 de junho de 2010. Aceito em versão revisada em 19 de outubro de 2010. 
ABSTRACT Objective. To test the validity and reliability of a self-efficacy scale for physical activity (PA) in Brazilian adults.

Psychometric properties of a

Methods. A self-efficacy scale was applied jointly with a multidimensional quesself-efficacy scale for physical tionnaire through face-to-face interviews with 1418 individuals (63.4\% women) aged $\geq 18$ years. The scale was submitted to validity (factorial and construct) and reliability analysis (internal consistency and temporal stability). A test-retest procedure was conducted with 74 individuals to evaluate temporal stability.

Results. Exploratory factor analyses revealed two independent factors: self-efficacy for walking and self-efficacy for moderate and vigorous PA (MVPA). Together, these two factors explained $65.4 \%$ of the total variance of the scale $(20.9 \%$ and $44.5 \%$ for walking and MVPA, respectively). Cronbach's alpha values were 0.83 for walking and 0.90 for MVPA, indicating high internal consistency. Both factors were significantly and positively correlated (rho $\geq 0.17, P<0.001$ ) with quality of life indicators (health perception, self-satisfaction, and energy for daily activities), indicating an adequate construct validity.

Conclusions. The scale's validity, internal consistency, and reliability were adequate to evaluate self-efficacy for PA in Brazilian adults.

Key words Motor Activity; exercise; psychometrics; self-efficacy; Brazil. 